\title{
Resolution of the more anteriorly positioned psoas muscle following correction of spinal sagittal alignment from spondylolisthesis: case report
}

\author{
Hasan R. Syed, MD, ${ }^{1}$ Kurt Yaeger, MD, ${ }^{2}$ and Faheem A. Sandhu, MD, PhD \\ 'Department of Neurosurgery, Medstar Georgetown University Hospital, Washington, DC; and 2Department of Neurosurgery, \\ Mount Sinai Hospital, New York, New York

\begin{abstract}
Several studies have described the radiographic, histological, and morphological changes to the paraspinal muscle in patients with chronic low-back pain due to degenerative diseases of the spine. Gross anatomical illustrations have shown that the psoas muscle lies lateral to the L4-5 vertebrae and subsequently thins and dissociates from the vertebral body at L5-S1 in a ventrolateral course. A "rising psoas" may influence the location of the lumbar plexus and result in transient neurological injury on lateral approach to the spine. It is postulated that axial back pain may be exacerbated by anatomical changes of paraspinal musculature as a direct result of degenerative spine conditions. To their knowledge, the authors present the first reported case of a more anteriorly positioned psoas muscle and its resolution following correction of spondylolisthesis in a 62-year-old woman. This case highlights the dynamic nature of degenerative spinal disorders and illustrates that psoas muscle position can be affected by sagittal balance. Normal anatomical positioning can be restored following correction of spinal alignment.
\end{abstract}

https://thejns.org/doi/abs/10.3171/2016.9.SPINE16324

KEY WORDS psoas; L4-5; lumbar; spondylolisthesis; lateral interbody fusion; sagittal balance

I NTERBODY fusion in the lumbar spine is a well-established strategy for treating degenerative disc disease and correcting spinal sagittal instability. ${ }^{8,25}$ Compared with traditional posterolateral lumbar fusion, interbody approaches are associated with higher rates of fusion in patients with degenerative spondylolisthesis who exhibit preoperative instability. ${ }^{9,25,30}$ The array of surgical approaches for interbody fusions in the lumbar spine allow for diverse therapeutic options, depending on the patient's individual anatomy and pathophysiology.

A lateral approach to lumbar interbody fusion (also described as direct lateral interbody fusion or extreme lateral interbody fusion) allows for the minimally invasive placement of a large intervertebral graft, with minimal destruction of osteoligamentous processes and retraction of neural elements., ${ }^{5,615,24}$ Furthermore, the lateral approach obviates the need for an approach surgeon and avoids the potentially morbid complications associated with an anterior approach. With a lower risk of significant bleeding and less damage to structural tissues, procedures in which a lateral exposure is used lead to reduced operation time, less postoperative pain, and shorter hospital stay. ${ }^{6,10,20,24}$
One potential complication of the transpsoas lateral interbody approach results from the surgical proximity to the psoas muscle and lumbar plexus, specifically at L4-5.,10,12,21,23 It has been reported that injury due to intraoperative traction, mechanical disruption, or thermal injury may contribute to postoperative iliopsoas and quadriceps muscle weakness or groin and thigh paresthesias in $2 \%-30 \%$ of cases, a majority of which resolve over time without additional intervention. ${ }^{4,23,24}$ Several anatomical studies have elucidated safer zones through which to approach the intervertebral space from a lateral perspective in an attempt to minimize lumbar plexus injury. ${ }^{1,2,11-13,29}$ Furthermore, intraoperative electromyography (EMG) and somatosensory evoked potentials are often used to avoid inadvertent nerve damage during the initial surgical approach. $16,34,35,37$

The psoas muscle originates from the transverse processes of T12-L5, courses ventrolaterally into the pelvis, and inserts into the lesser trochanter of the femur. The psoas muscle belly diameter is largest around L5-S1, and thins substantially as it enters the pelvis, where it dissociates from the vertebral bodies prior to its insertion point 


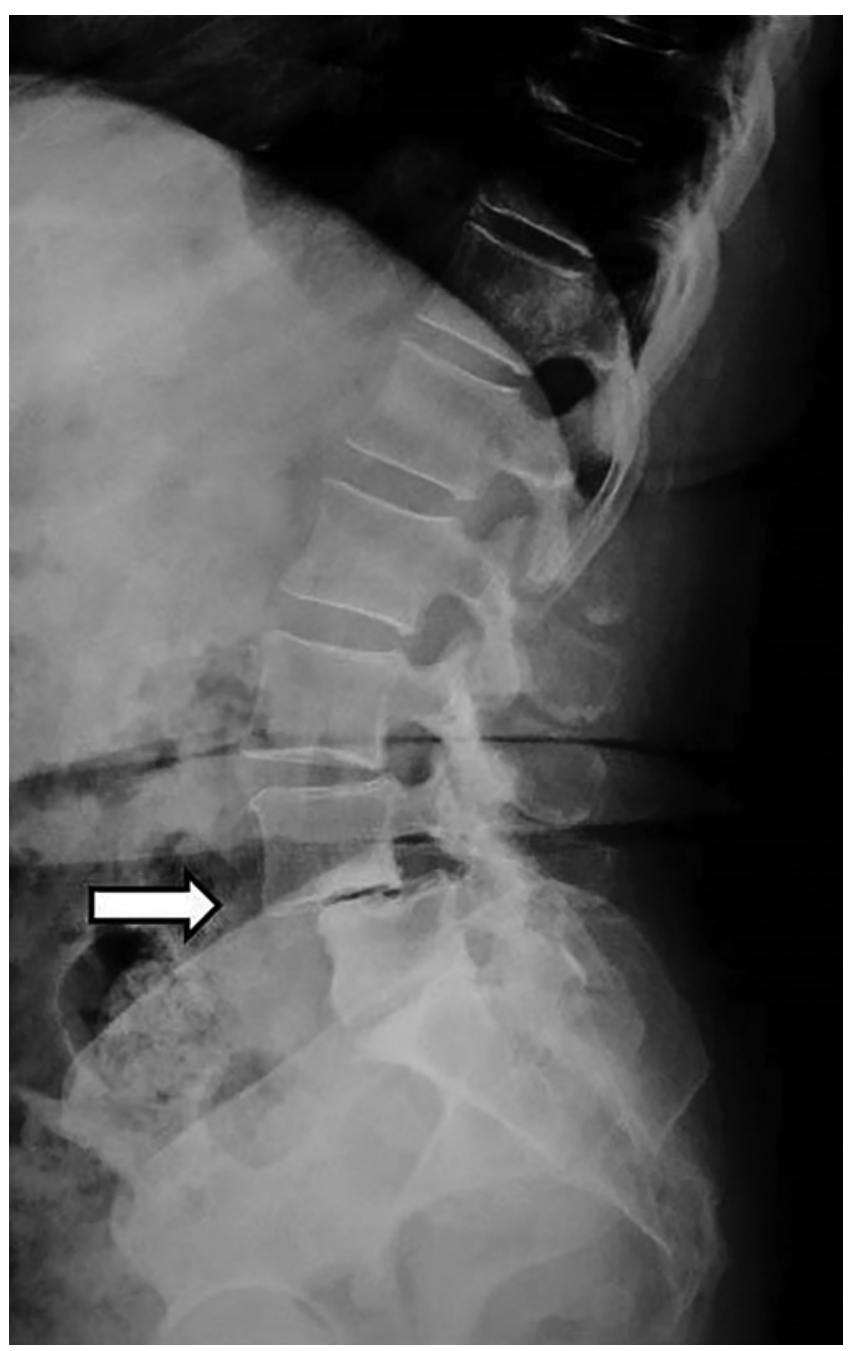

FIG. 1. Standing lateral radiograph showing a Grade $2 \mathrm{~L} 4-5$ spondylolisthesis (arrow).

caudally. Although anatomical studies have illustrated relatively safe corridors for lateral transpsoas approaches, these typical surgical strategies may be less valuable in patients with variable or asymmetrical paraspinal musculature, as has been associated with degenerative spinal diseases. ${ }^{17-19,31,33,39}$ A variable psoas muscle anatomy is especially concerning because of the intrinsic relationship between the muscle belly and the lumbar plexus.

The previously reported "rising psoas sign" is a radiological finding in which the psoas muscle is located ventral to the vertebral body, rather than lateral, and may indicate an increased risk of nerve injury with a lateral surgical approach, due to ventral displacement of the lumbar plexus. ${ }^{38}$ This finding was initially observed retrospectively on MRI in 3 patients undergoing lateral interbody fusion procedures for L4-5 spondylolisthesis whose surgeries were aborted because of robust EMG signals during the initial approach. To our knowledge, we present the first reported case of anatomical resolution of a more anteriorly displaced psoas muscle following correction of spondylolisthesis.

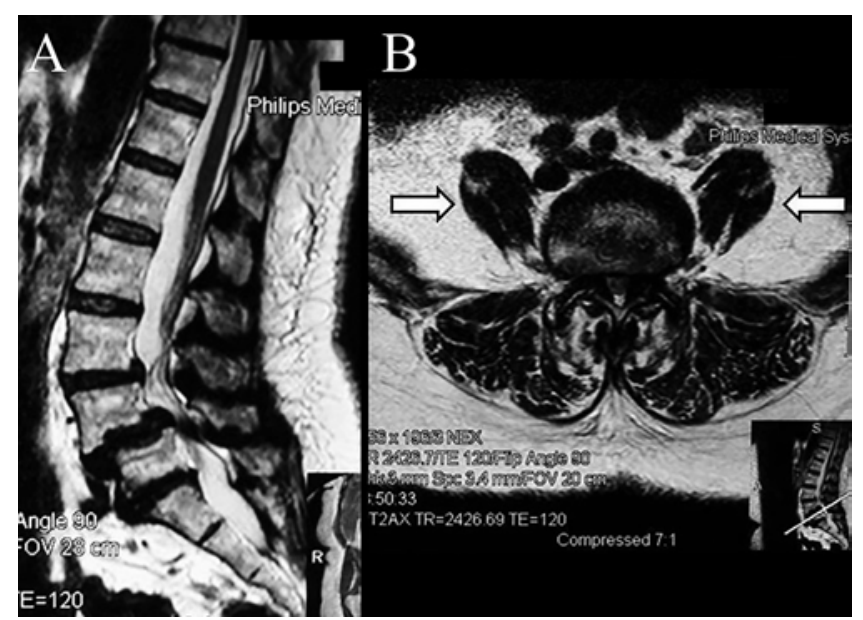

FIG. 2. Sagittal (A) and axial (B) MR images showing a Grade 2 L4-5 spondylolisthesis. The psoas muscle can be seen rising away from vertebral body bilaterally (arrows).

\section{Case Report}

History and Examination

A 62-year-old woman presented with several months of progressively worsening low-back pain, with radiation down the right leg that failed to improve with conservative measures. Neurological examination demonstrated mild right extensor hallucis longus weakness (Grade 4+/5). Imaging was significant for Grade 2 spondylolisthesis at L4-5 on lateral radiography (Fig. 1), as well as a broad disc protrusion at L4-5 causing foraminal stenosis and moderate spinal canal compromise on MRI. Bilateral psoas muscles rising ventrally from the vertebral bodies were also noted on preoperative MRI (Fig. 2).

\section{Operation and Technique}

The patient underwent an L4-5 lateral interbody fusion (Ravine Lateral Access System, K2M), followed by percutaneous placement of pedicle screws at L4-5 (Romeo 2 K-Wireless, Spineart). Neuromonitoring, including EMG recordings, were planned, and the needles were inserted in the usual fashion. This included monitoring L-2 and L-3 (the rectus femoris muscle), L-3 and L-4 (the vastus medialis muscle), L-4 (the anterior tibialis muscle), L-5 and S-1 (peroneus longus), and S-1 and S-2 (gastrocnemius) for all cases. Baseline measures were obtained, and stimulation was provided multiple times during decompression and insertion of the lateral device. There were no intraoperative complications.

The patient was placed in the standard lateral decubitus position with her left side up. An axillary roll was placed under the right axilla, and all pressure points were padded. The chest and legs were secured to the operating table with tape. The table was subsequently broken at the level of the iliac crest to provide greater access to the level of interest. Fluoroscopy was used to localize the L4-5 level (Fig. $3 \mathrm{~A})$. After the area was prepared and draped in the usual fashion, a $2.5-\mathrm{cm}$ skin incision directly over the disc space of interest was made, followed by dissection of the subcutaneous soft tissue. Then, the oblique muscles were split 

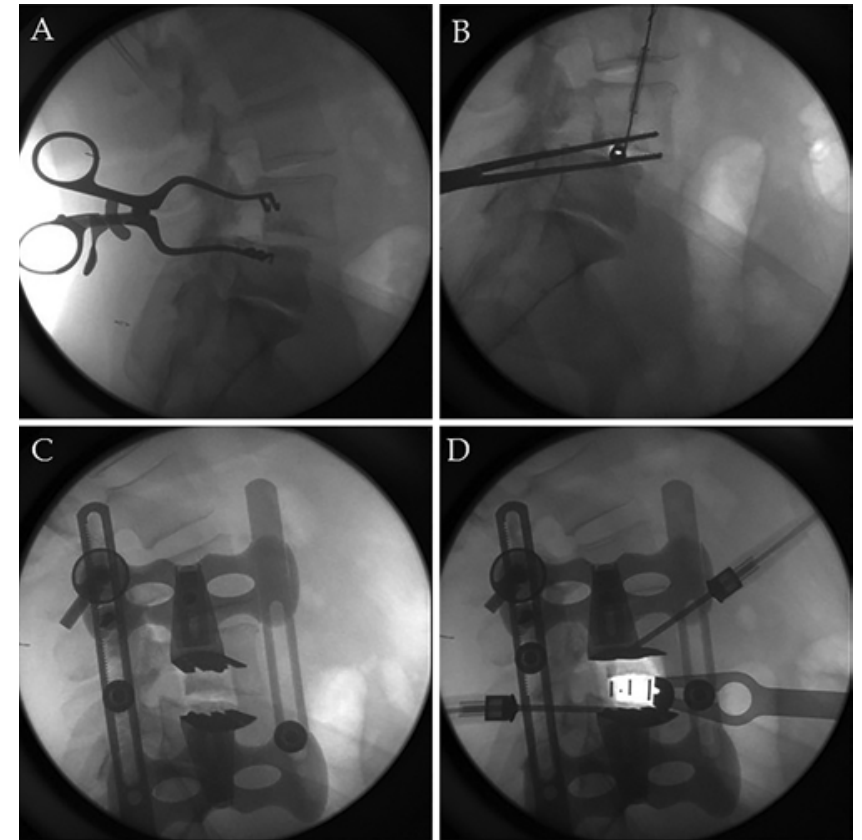

FIG. 3. Intraoperative lateral fluoroscopic images showing access to the L4-5 disc space. An incision is made, centered at L4-5 level (A). The first dilator is inserted under the guidance of neuromonitoring and docked near the midpoint of the disc space (B). The Ravine Lateral Access Retractor is docked at the center of the L4-5 disc space (C). Final fluoroscopic image showing the dual blade retractor with rigid fixation to the spine and insertion of the lateral interbody cage (D).

bluntly, and the retroperitoneal space was entered. Under direct visualization, the preperitoneal fat was swept forward, uncovering the psoas muscle. At this point, the first dilator was placed on the surface of the psoas muscle and passed through using continuous neuromonitoring with the aim of docking in the midpoint of the disc space (Fig. 3B). No EMG activity was noted, and a guidewire was placed through the dilator to secure the position to the disc space. Two sequential oblong dissectors were then passed over the guidewire in line with the psoas muscle fibers and rotated circumferentially to help release the psoas muscle from the annulus of the disc. Continuous EMG monitoring stimulated throughout the base of both dissectors was performed to ensure protection of the lumbar plexus. The Ravine Lateral Access retractor was passed over the guidewire in line with the psoas muscle fibers, turned $90^{\circ}$, expended, and secured to the vertebral bodies with fixation screws. Proper positioning of the retractor was confirmed on fluoroscopic imaging (Fig. 3C). Direct monopolar stimulation of the exposed disc area with a minimal stimulation threshold further confirmed a safe working zone. A thorough discectomy was then performed in the traditional manner, and the contralateral annulus was released. After appropriate endplate preparation, an interbody cage was then packed with bone graft material (bone morphogenetic protein; Infuse, Medtronic) and inserted into the disc space. Satisfactory placement of the cage was confirmed using anteroposterior and lateral fluoroscopy (Fig. 3D). The working channel was then removed, and the incision was closed in the standard manner.

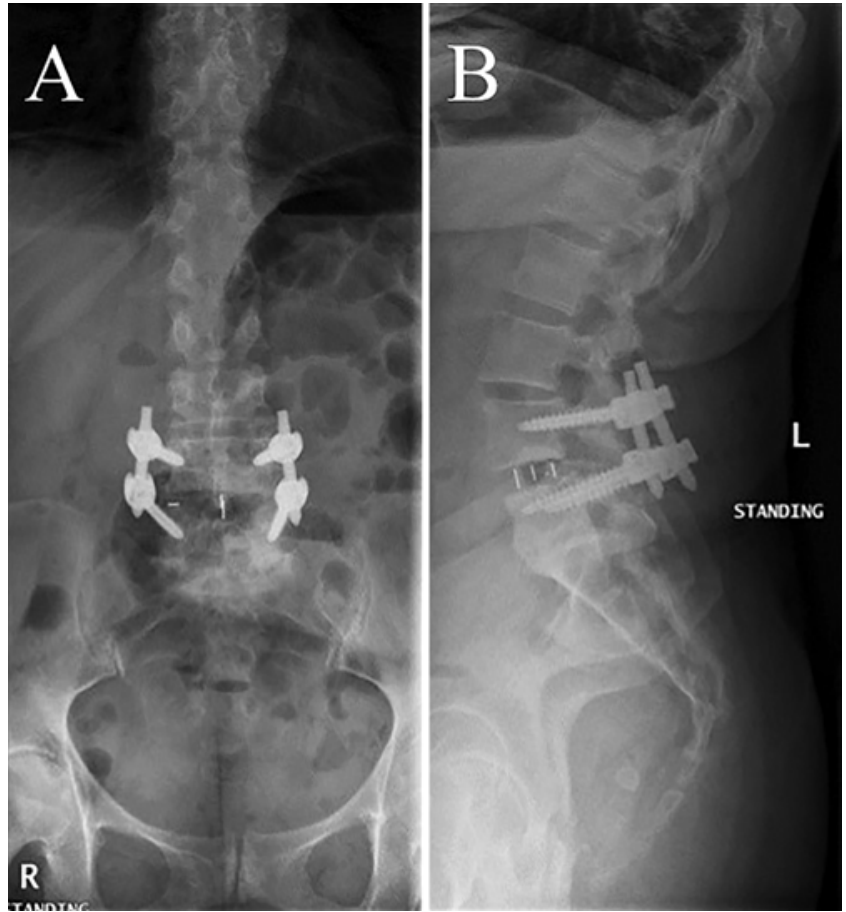

FIG. 4. Postoperative anteroposterior (A) and lateral (B), radiographs, demonstrating L4-5 lateral interbody fusion with percutaneous pedicle screws.

\section{Postoperative Course}

The patient recovered well from her procedure, with immediate resolution of her radicular symptoms. She was discharged from the hospital on postoperative Day 2. She experienced complete resolution of her back and leg symptoms by 3 months. Postoperative radiography demonstrated anatomical reduction of L4-5 spondylolisthesis (Fig. 4). Follow-up MR imaging at 3 months showed resolution of foraminal and central canal stenosis caused by indirect decompression. Upon review of the paraspinal musculature, the psoas muscles were observed in their anatomical position, lateral to the lumbar vertebral bodies, in contrast to their preoperative ventral location (Fig. 5).

\section{Discussion}

Minimally invasive lumbar interbody fusion is a safe, effective alternative to open methods of lumbar fusion, and is currently used to treat a wide variety of lumbar spinal pathology, including degenerative disc disease, spondylolisthesis, and scoliosis. ${ }^{9,15,20,32}$ Clinical outcomes following interbody fusion are comparable to traditional procedures, yet these minimally invasive operations allow for smaller incisions and minimal muscle dissection, leading to less postoperative pain, fewer surgical site infections, and decreased muscle denervation. ${ }^{6}$

Several approaches are commonly used to access the intervertebral space, each with varying indications depending on individual patient anatomy and pathology, as well as surgeon experience and preference. The lateral approach, commonly performed as either the direct or ex- 

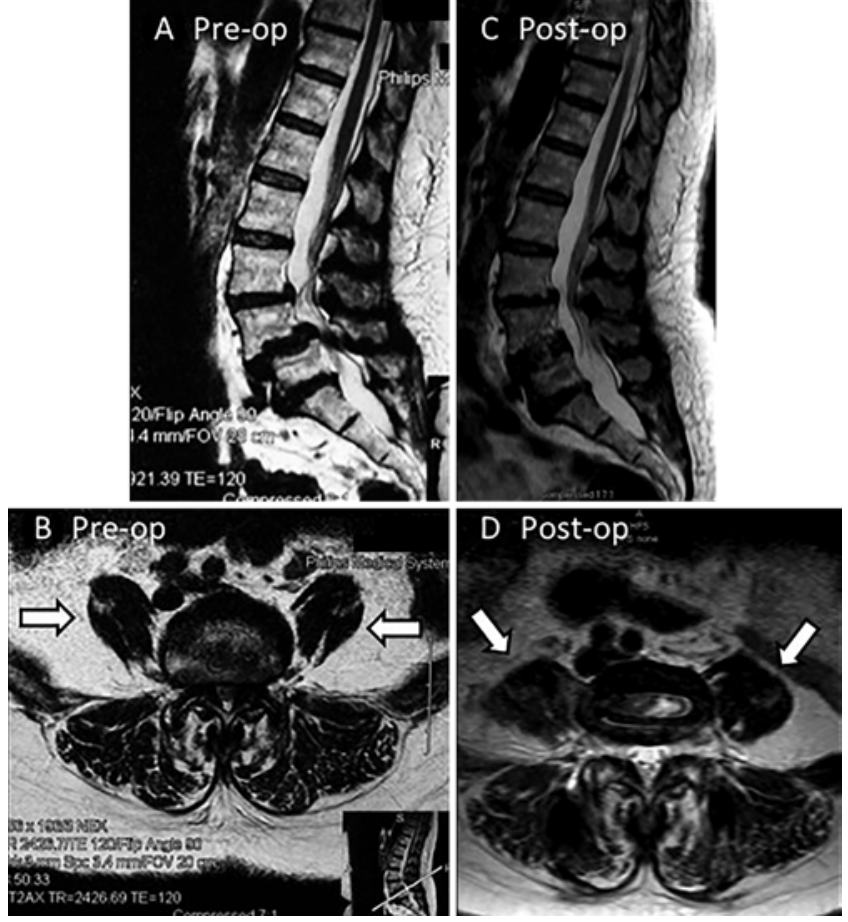

FIG. 5. Comparison of preoperative sagittal (A) and axial (B) MR images and follow-up postoperative sagittal (C) and axial (D) MR images demonstrating a reduction of spondylolisthesis. The psoas muscle can be seen lying lateral to the vertebra at L4-5 in its normally described anatomical position in contrast to the preoperative images (arrows).

treme lateral interbody fusion, allows for the placement of a large intervertebral graft with relative preservation of spinal osteoligamentous integrity., ${ }^{5,15,24,27}$ By using a lateral, retroperitoneal, transpsoas trajectory, the anterior and posterior spinal elements are conserved. One significant consequence of traversing this anatomical corridor is potential injury to nerves of the lumbar plexus, which lies ventral to the psoas muscle most significantly at L4-5. Iatrogenic lumbar nerve injury, caused by initial dilator placement or subsequent manipulation of the retractor, may contribute to motor weakness in the hip flexors or knee extensors, or sensory deficits, including paresthesias, numbness, or radicular pain, throughout the thigh. Although complication rates vary depending on the individual study, between $0.7 \%$ and $54.9 \%$ for muscle weakness and $12 \%$ and $25 \%$ for sensory deficits, most postoperative deficits are transitory, resolving within weeks to months. . $^{60,21,32}$

Since the course of the lumbar plexus is inexorably linked to psoas muscle anatomy, any variation to the paraspinal musculature may lead to inadvertent nerve injury, despite utilizing commonly practiced surgical approaches. Several studies have investigated the relationship between anatomical variations in paraspinal musculature and spinal balance in patients with degenerative lumbosacral diseases, including scoliosis and spondylolisthesis.

The role of paraspinal muscle asymmetry as a cause of, or a compensatory mechanism for, degenerative spinal imbalance remains incompletely clarified. In the case of degenerative scoliosis, progressive coronal instability may be theorized to cause stretching and thinning of the paraspinal muscles on the convex side, and consequent shortening and thickening of the concave musculature. However, in a retrospective study of the cross-sectional area (CSA) of paravertebral musculature in patients with degenerative scoliosis, Kim and coauthors observed that the CSAs of the psoas and multifidus muscles were significantly greater on the convex side of the deformity, compared with the concave side, whereas the degree of muscle atrophy, as indicated by fatty infiltration, was consistent bilaterally. ${ }^{19}$ These findings allude to compensatory paraspinal muscle hypertrophy as a consequence of progressive coronal imbalance, a conclusion that fits with the long-standing theory that the psoas and paraspinal muscles are dynamic stabilizers of the spinal column. ${ }^{26,28}$ Another retrospective cohort of patients with degenerative lumbar scoliosis and spinal stenosis was similarly observed by Shafaq et al. to have asymmetric paravertebral muscles, with smaller CSAs and greater fatty infiltration in muscles along the concavity. ${ }^{33}$ These atrophic concave muscles appeared to be histologically denervated, with decreased fiber sizes and fewer nuclei, and suggest a possible role of lumbar radiculopathy, as a consequence of spinal imbalance, in the mechanism of asymmetrical paraspinal muscle degeneration. This pathophysiological response has been shown to occur similarly in patients with unilateral back pain and monosegmental degenerative disc disease, in whom the paraspinal muscle CSA is significantly smaller on the symptomatic side. ${ }^{31}$ The fact that this finding can occur without structural deformity (i.e., scoliosis or spondylolisthesis), indicates the secondary, rather than causative, nature of asymmetrical muscle atrophy on spinal imbalance.

Paraspinal muscle asymmetry has also been investigated in patients with spinal sagittal imbalance. When comparing 108 patients with either lumbar degenerative kyphosis or chronic low-back pain, Kang and colleagues observed a significant reduction in the cross-sectional area of the psoas, erector spinae, and multifidus in patients with a kyphotic deformity. ${ }^{17}$ Furthermore, the paraspinal musculature in patients with structural disease was associated with a greater degree of fatty infiltration, an indicator of severe muscular atrophy, occurring in 54\% of patients (compared with $7 \%$ of patients without structural deformity). These data were echoed by a comparable study by Lee and coauthors, again illustrating a decreased CSA and greater fat infiltration of the paraspinal muscles in patients with degenerative lumbar kyphosis than in healthy controls. ${ }^{22}$ Hyun et al. observed similar atrophic muscular changes, both in terms of reduced CSA and enhanced fatty infiltration in patients with degenerative lumbar kyphosis, most significantly within the multifidus and erector spinae at the levels of L-4 and L-5. ${ }^{14}$ The selective atrophy of these posterior muscle groups in association with lumbar kyphosis highlights the importance of paraspinal muscles in maintaining sagittal balance. Wang and colleagues examined 149 patients with degenerative spondylolisthesis and similarly observed a greater degree of multifidus muscle atrophy compared with controls. ${ }^{39}$ In contrast, however, the erector spinae muscles were significantly larger in 
patients with sagittal imbalance than in patients without deformity, possibly representing a compensatory mechanism to maintain sagittal stability.

In the present case, preoperative images in a 62-year-old patient with L4-5 spondylolisthesis and progressive lowback pain highlighted the so-called "rising psoas sign," an imaging finding that has been associated with intraoperative EMG disturbances, and a theoretical risk of nerve damage when proceeding with a lateral transpsoas lumbar interbody fusion procedure. We believe that the risk of nerve injury can be mitigated by using the aforementioned technique. The Ravine Lateral Access System uses dual flat blades instead of the standard tubular retractor with 3 channels in the blades to help with ideal positioning of the retractor over the guidewire (Fig. 6). For example, the guidewire can be placed very anteriorly on the disc space (just behind the anterior longitudinal ligament), and, using the anterior-most channel on the retractor blade, it can still provide excellent exposure of the disc space of interest. The advantages of this technique include a reproducible working corridor at the level of interest that respects psoas muscle anatomy. Three critical steps help provide the surgeon with the safest and most optimal positioning for the retractor system. First, the psoas muscle is split longitudinally, which is in line with the direction of the muscle fibers, using serial oblong dissectors and the dual blades of the retractor. Second, neuromonitoring with EMG during initial dilation and subsequent dissection steps helps avoid the lumbar plexus and safely place the retractor. Unlike tubular retractor systems that need to be placed more posteriorly on the disc space to allow adequate room to open the retractor to complete the discectomy, the Ravine dual blade system may be deployed after placing the initial guidewire anywhere on the disc space that is free and clear of the lumbar plexus. The nerve and muscle are swept away by the retractor to provide a safe working corridor. Finally, after placement of the dual-blade retractor system over the level of interest and opening it to expose the disc space, the position is maintained by affixing the retractor to the vertebral bodies with screws. Then, direct monopolar stimulation with minimal triggered EMG activity allows further confirmation of a safe entry zone. Consequently, despite the abnormal psoas anatomy, the rationale to pursue the lateral transpsoas approach in this case was based on our experience that the risk of nerve injury is low with this retractor system. After undergoing correction of sagittal imbalance via an L4-5 lateral interbody fusion and transcutaneous pedicle screw placement at L-4 and L-5 bilaterally, the patient's symptoms resolved, and the aberrant psoas muscle anatomy returned to a normal position directly lateral to the spinal column.

Patients with degenerative spinal deformity who have undergone corrective surgery may experience postoperative changes in paravertebral musculature. In a comparison of minimally invasive versus conventional, open posterior interbody fusion, atrophy of the multifidus muscle was observed in all patients, as indicated by the muscle CSAs and T2 signal intensity (fatty infiltration). ${ }^{7}$ However, less mulitfidus atrophy occurred in patients undergoing minimally invasive posterior lumbar interbody fusion (PLIF). These data were confirmed by a similar study that com-

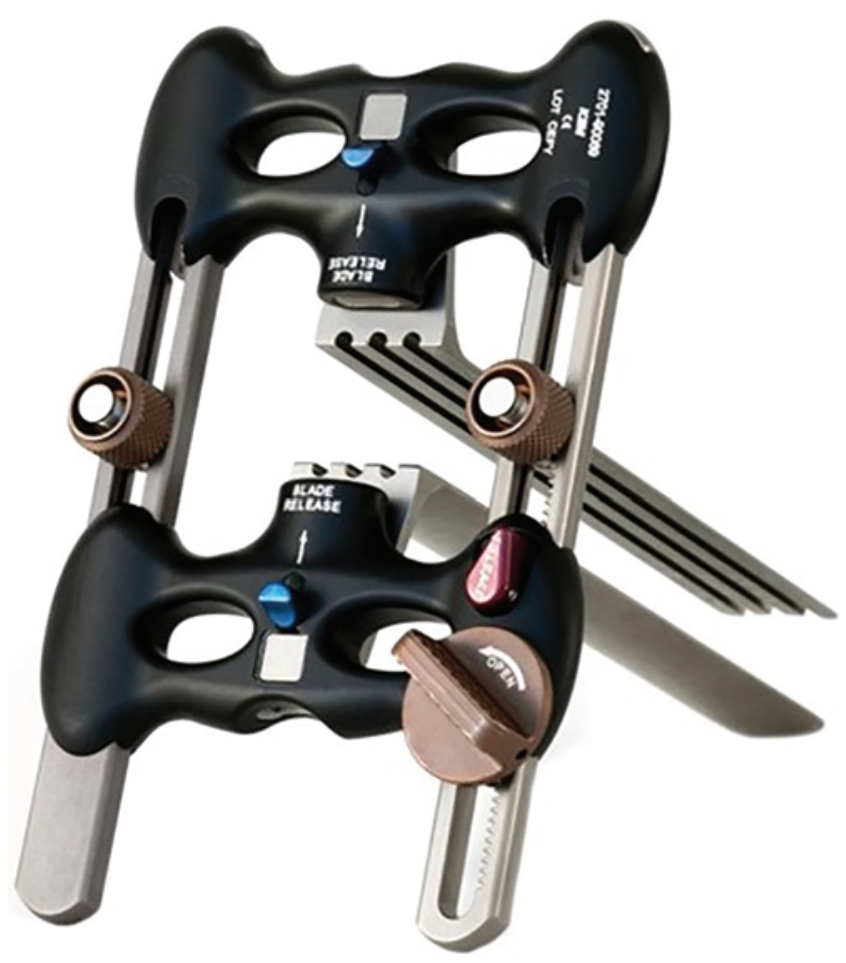

FIG. 6. The Ravine Lateral Access Retractor uses dual flat blades with options for a third and fourth blade. Three channels in each blade allow for adjustment on retractor position depending on the initial docking point. The advantages of this system include dissection and retraction of the psoas muscle in a more anatomical manner and optimum adaptability to the patient's anatomy. Copyright K2M. Published with permission. Figure is available in color online only.

pared patients undergoing open PLIF versus a mini-open PLIF, in which the more minimally invasive procedure allowed better preservation of the multifidus muscle. ${ }^{36}$

\section{Conclusions}

We describe the first case in the literature of a patient with spinal sagittal deformity and aberrant paravertebral musculature on preoperative imaging who experienced resolution of both symptoms and anomalous musculature after correction of the spondylolisthesis. This observation highlights the association between the psoas and other paravertebral muscles and spinal balance. Whether a causative or compensatory mechanism, this relationship remains to be clarified. This case highlights the dynamic nature of degenerative spinal disorders and illustrates that psoas muscle position can be affected by sagittal balance. Normal anatomical positioning can be restored following correction of spinal alignment.

\section{References}

1. Banagan K, Gelb D, Poelstra K, Ludwig S: Anatomic mapping of lumbar nerve roots during a direct lateral transpsoas approach to the spine: a cadaveric study. Spine (Phila Pa 1976) 36:E687-E691, 2011

2. Benglis DM, Vanni S, Levi AD: An anatomical study of the lumbosacral plexus as related to the minimally invasive 
transpsoas approach to the lumbar spine. J Neurosurg Spine 10:139-144, 2009

3. Bina RW, Zoccali C, Skoch J, Baaj AA: Surgical anatomy of the minimally invasive lateral lumbar approach. J Clin Neurosci 22:456-459, 2015

4. Cahill KS, Martinez JL, Wang MY, Vanni S, Levi AD: Motor nerve injuries following the minimally invasive lateral transpsoas approach. J Neurosurg Spine 17:227-231, 2012

5. Dahdaleh NS, Smith ZA, Snyder LA, Graham RB, Fessler RG, Koski TR: Lateral transpsoas lumbar interbody fusion: outcomes and deformity correction. Neurosurg Clin N Am 25:353-360, 2014

6. Dakwar E, Cardona RF, Smith DA, Uribe JS: Early outcomes and safety of the minimally invasive, lateral retroperitoneal transpsoas approach for adult degenerative scoliosis. Neurosurg Focus 28(3):E8, 2010

7. Fan S, Hu Z, Zhao F, Zhao X, Huang Y, Fang X: Multifidus muscle changes and clinical effects of one-level posterior lumbar interbody fusion: minimally invasive procedure versus conventional open approach. Eur Spine J 19:316-324, 2010

8. Fritzell P, Hägg O, Wessberg P, Nordwall A: 2001 Volvo Award Winner in Clinical Studies: Lumbar fusion versus nonsurgical treatment for chronic low back pain: a multicenter randomized controlled trial from the Swedish Lumbar Spine Study Group. Spine (Phila Pa 1976) 26:2521-2534, 2001

9. Gandhoke GS, Kasliwal MK, Smith JS, Nieto J, Ibrahimi $D$, Park P, et al: A multi-center evaluation of clinical and radiographic outcomes following high-grade spondylolisthesis reduction and fusion. Clin Spine Surg [epub ahead of print], 2016

10. Graham RB, Wong AP, Liu JC: Minimally invasive lateral transpsoas approach to the lumbar spine: pitfalls and complication avoidance. Neurosurg Clin N Am 25:219-231, 2014

11. Guérin P, Obeid I, Gille O, Bourghli A, Luc S, Pointillart V, et al: Safe working zones using the minimally invasive lateral retroperitoneal transpsoas approach: a morphometric study. Surg Radiol Anat 33:665-671, 2011

12. Houten JK, Alexandre LC, Nasser R, Wollowick AL: Nerve injury during the transpsoas approach for lumbar fusion. J Neurosurg Spine 15:280-284, 2011

13. Hu WK, He SS, Zhang SC, Liu YB, Li M, Hou TS, et al: An MRI study of psoas major and abdominal large vessels with respect to the X/DLIF approach. Eur Spine J 20:557-562, 2011

14. Hyun SJ, Bae CW, Lee SH, Rhim SC: Fatty degeneration of paraspinal muscle in patients with the degenerative lumbar kyphosis: a new evaluation method of quantitative digital analysis using MRI and CT scan. Clin Spine Surg [epub ahead of print], 2016

15. Isaacs RE, Hyde J, Goodrich JA, Rodgers WB, Phillips FM: A prospective, nonrandomized, multicenter evaluation of extreme lateral interbody fusion for the treatment of adult degenerative scoliosis: perioperative outcomes and complications. Spine (Phila Pa 1976) 35 (26 Suppl):S322-S330, 2010

16. Jahangiri FR, Sherman JH, Holmberg A, Louis R, Elias J, Vega-Bermudez F: Protecting the genitofemoral nerve during direct/extreme lateral interbody fusion (DLIF/XLIF) procedures. Am J Electroneurodiagn Technol 50:321-335, 2010

17. Kang CH, Shin MJ, Kim SM, Lee SH, Lee CS: MRI of paraspinal muscles in lumbar degenerative kyphosis patients and control patients with chronic low back pain. Clin Radiol 62:479-486, 2007

18. Kepler CK, Bogner EA, Herzog RJ, Huang RC: Anatomy of the psoas muscle and lumbar plexus with respect to the surgical approach for lateral transpsoas interbody fusion. Eur Spine J 20:550-556, 2011

19. Kim H, Lee CK, Yeom JS, Lee JH, Cho JH, Shin SI, et al:
Asymmetry of the cross-sectional area of paravertebral and psoas muscle in patients with degenerative scoliosis. Eur Spine J 22:1332-1338, 2013

20. Knight RQ, Schwaegler P, Hanscom D, Roh J: Direct lateral lumbar interbody fusion for degenerative conditions: early complication profile. J Spinal Disord Tech 22:34-37, 2009

21. Le TV, Burkett CJ, Deukmedjian AR, Uribe JS: Postoperative lumbar plexus injury after lumbar retroperitoneal transpsoas minimally invasive lateral interbody fusion. Spine (Phila Pa 1976) 38:E13-E20, 2013

22. Lee JC, Cha JG, Kim Y, Kim YI, Shin BJ: Quantitative analysis of back muscle degeneration in the patients with the degenerative lumbar flat back using a digital image analysis: comparison with the normal controls. Spine (Phila Pa 1976) 33:318-325, 2008

23. Lykissas MG, Aichmair A, Hughes AP, Sama AA, Lebl DR, Taher F, et al: Nerve injury after lateral lumbar interbody fusion: a review of 919 treated levels with identification of risk factors. Spine J 14:749-758, 2014

24. Moller DJ, Slimack NP, Acosta FL Jr, Koski TR, Fessler RG, Liu JC: Minimally invasive lateral lumbar interbody fusion and transpsoas approach-related morbidity. Neurosurg Focus 31(4): E4, 2011

25. Mummaneni PV, Dhall SS, Eck JC, Groff MW, Ghogawala Z, Watters WC III, et al: Guideline update for the performance of fusion procedures for degenerative disease of the lumbar spine. Part 11: interbody techniques for lumbar fusion. J Neurosurg Spine 21:67-74, 2014

26. Nachemson A: The possible importance of the psoas muscle for stabilization of the lumbar spine. Acta Orthop Scand 39:47-57, 1968

27. Ozgur BM, Aryan HE, Pimenta L, Taylor WR: Extreme lateral interbody fusion (XLIF): a novel surgical technique for anterior lumbar interbody fusion. Spine J 6:435-443, 2006

28. Panjabi MM: The stabilizing system of the spine. Part I. Function, dysfunction, adaptation, and enhancement. J Spinal Disord 5:383-389, 397, 1992

29. Park DK, Lee MJ, Lin EL, Singh K, An HS, Phillips FM: The relationship of intrapsoas nerves during a transpsoas approach to the lumbar spine: anatomic study. J Spinal Disord Tech 23:223-228, 2010

30. Park P, Wang MY, Lafage V, Nguyen S, Ziewacz J, Okonkwo $\mathrm{DO}$, et al: Comparison of two minimally invasive surgery strategies to treat adult spinal deformity. J Neurosurg Spine 22:374-380, 2015

31. Ploumis A, Michailidis N, Christodoulou P, Kalaitzoglou I, Gouvas G, Beris A: Ipsilateral atrophy of paraspinal and psoas muscle in unilateral back pain patients with monosegmental degenerative disc disease. Br J Radiol 84:709-713, 2011

32. Rodgers WB, Gerber EJ, Patterson J: Intraoperative and early postoperative complications in extreme lateral interbody fusion: an analysis of 600 cases. Spine (Phila Pa 1976) 36:2632,2011

33. Shafaq N, Suzuki A, Matsumura A, Terai H, Toyoda H, Yasuda $\mathrm{H}$, et al: Asymmetric degeneration of paravertebral muscles in patients with degenerative lumbar scoliosis. Spine (Phila Pa 1976) 37:1398-1406, 2012

34. Silverstein J, Mermelstein L, DeWal H, Basra S: Saphenous nerve somatosensory evoked potentials: a novel technique to monitor the femoral nerve during transpsoas lumbar lateral interbody fusion. Spine (Phila Pa 1976) 39:1254-1260, 2014

35. Tohmeh AG, Rodgers WB, Peterson MD: Dynamically evoked, discrete-threshold electromyography in the extreme lateral interbody fusion approach. J Neurosurg Spine 14:3137,2011

36. Tsutsumimoto T, Shimogata M, Ohta H, Misawa H: Miniopen versus conventional open posterior lumbar interbody fusion for the treatment of lumbar degenerative spondylo- 
listhesis: comparison of paraspinal muscle damage and slip reduction. Spine (Phila Pa 1976) 34:1923-1928, 2009

37. Uribe JS, Vale FL, Dakwar E: Electromyographic monitoring and its anatomical implications in minimally invasive spine surgery. Spine (Phila Pa 1976) 35 (26 Suppl):S368-S374, 2010

38. Voyadzis JM, Felbaum D, Rhee J: The rising psoas sign: an analysis of preoperative imaging characteristics of aborted minimally invasive lateral interbody fusions at L4-5. J Neurosurg Spine 20:531-537, 2014

39. Wang G, Karki SB, Xu S, Hu Z, Chen J, Zhou Z, et al: Quantitative MRI and X-ray analysis of disc degeneration and paraspinal muscle changes in degenerative spondylolisthesis. J Back Musculoskeletal Rehabil 28:277-285, 2015

\section{Disclosures}

Dr. Sandhu receives royalties for use of the Ravine retractor from
$\mathrm{K} 2 \mathrm{M}$. The other authors report no conflict of interest concerning the materials or methods used in this study or the findings specified in this paper.

\section{Author Contributions}

Conception and design: Sandhu, Syed. Acquisition of data: Sandhu, Syed. Analysis and interpretation of data: Sandhu, Syed. Drafting the article: all authors. Critically revising the article: Sandhu, Syed. Reviewed submitted version of manuscript: all authors. Approved the final version of the manuscript on behalf of all authors: Sandhu. Administrative/technical/material support: Sandhu, Syed. Study supervision: Sandhu, Syed.

\section{Correspondence}

Faheem A. Sandhu, Department of Neurosurgery, Medstar Georgetown University Hospital, Pasquerilla Healthcare Center, Seventh Fl., 3800 Reservoir Rd. NW, Washington, DC 20007. email: fasandhu@aol.com. 